\title{
A Discrete Stochastic Process for Coverage Analysis of Autonomous UAV Networks
}

\author{
Evşen Yanmaz*, Carmelo Costanzo*†, Christian Bettstetter*+, and Wilfried Elmenreich* \\ ${ }^{*}$ Mobile Systems Group, Institute of Networked and Embedded Systems, University of Klagenfurt, Austria \\ $\dagger$ Dipartimento di Elettronica Informatica e Sistemistica (DEIS), University of Calabria, Rende, Italy \\ ${ }^{+}$Lakeside Labs GmbH, Klagenfurt, Austria
}

\begin{abstract}
Networked unmanned aerial vehicles (UAVs) have found an increasing number of applications in recent years. In this work, we provide an analytical method to evaluate the sensor coverage performance of a UAV network, where the individual UAVs can work independently or cooperatively, respectively, to achieve a common goal. More specifically, we propose a stochastic model in terms of a Markov chain including approximations for its parameters. Studying several scenarios using the Markov chain as well as simulations, we investigate the impact of network size and area size on the achieved coverage. While the Markovbased analysis is an approximation, the results are still in good agreement with the simulations.
\end{abstract}

Index Terms-UAV networks, wireless sensor networks, mobility, coverage

\section{INTRODUCTION}

This paper considers a network of unmanned aerial vehicles (UAVs). Each UAV is equipped with a certain kind of onboard sensor, for example, a camera or a different sensor, taking snapshots of the ground area. The general aim of the UAV network is to explore a given area, i.e., to somehow "cover" this area using several snapshots. Such a goal is relevant to several applications: target or event detection and tracking in an unknown area; monitoring geographically inaccessible or dangerous areas (e.g., wildfire, volcano), or assisting emergency personnel in case of disasters. Recently, several researchers in the domains of robotics and mobile networking have focused on designing such UAV networks. Research takes place in various areas, e.g., control engineering, communication networking, mission planning, and image processing. A UAV is sometimes also called drone.

Our objective is to provide a simple analytical method to evaluate the performance of different UAV mobility patterns in terms of their coverage distribution. To this end, we propose a stochastic model using a Markov chain. The states are the locations of drones, and the transitions are determined by the mobility model of interest. Such a model can easily be created for independent mobility models, such as the random walk and random direction. However, for a cooperative network, in which each drone decides where to move based on the information received from other drones in its communication range, creating a simple Markov model is not straightforward. Therefore, in this work, in addition to providing the necessary transition probabilities for random walk and random direction, we also propose an approximation to these probabilities for a cooperative network. While we choose intuitive rules for the movement paths when two or more drones "meet each other," the proposed model can be extended such that other rules can be incorporated. We show the validity of the proposed tool by comparing the analytical results with simulations for several scenarios with different network sizes as well as different geographical area sizes. With this tool, steadystate coverage distribution, average and full coverage times for random walk, direction and cooperative mobility models are evaluated, where the analysis and simulation are in good agreement.

The remainder of the paper is organized as follows. In Section II background on analysis of mobility models and coverage problem in wireless networks and robotics is summarized. The proposed analytical method is presented in Section III. Transition probability matrices for independent and cooperative mobility models are provided in Section IV. Results are given in Section $\mathrm{V}$ and the paper is concluded in Section VI.

\section{RELATED WORK}

Several mobility models for autonomous agents have been proposed recently. Some of these are synthetic like the random walk and random direction others are realistic and, all of them, are used mainly to describe the movement of the users in a given environment. In the UAV domain, such models are good for comparison of different approaches, but can give incorrect results when UAVs are performing cooperative tasks [1].

Recently, several research works have shown how mobility can increase throughput [2], energy efficiency [3], coverage [4], and other network parameters. Therefore, the analysis of mobility models has become a highlight to design the mobility of the nodes in a way to improve the network performance. A tool to analyze mobility models is proposed in [5], where the authors model random waypoint-like models as a renewal process to show the steady-state distribution of the speed, while a spatial analysis of different mobility models is provided in [6].

Also, the robotics community is involved in problems related to the coverage of an unknown environment also known as the sweeping problem [7]. Basically, the problem can either be solved by providing abilities for localization and map building first or by directly deriving an algorithm that performs sweeping without explicit mapping of the area. In [8], an exploration algorithm that allows multiple robots to 
cooperatively sweep an area is described. Instead of a measure of coverage, the authors measure the average event detection time for evaluating their algorithm. In addition, coverage problem is sometimes referred to as mapping of an unknown environment and there are useful methods summarized next for motion control and navigation, but they are not directly applicable to coverage or sweeping analysis. In [9], the authors introduce the concept of occupancy grid that is a stochastic estimate of the obstacle coverage of the cells obtained by sensing the environment and can be used for both mapping and navigation. Another technique proposed in [10] permits not only the mapping, but also the localization of the robot on the map.

In this work, we focus on the sweeping of an unknown area by probabilistic mobility patterns. Our contribution is to provide an analytical tool to represent existing and possibly new mobility models. We achieve this by providing transition probabilities among positions on a discrete grid and we give a means to compare different mobility patterns in terms of achieved area coverage at a given time or, even better, to design a new model that is able to achieve a desired coverage.

\section{Markov Chain and Coverage Metrics}

\section{A. Markov Chain}

We introduce a discrete-time, discrete-value stochastic process that can be used to analyze the coverage performance of a UAV network. Nodes can operate independently or in a cooperative manner. The system area is modeled as a twodimensional lattice where drones move from one grid point to another in each time step. We assume that a drone can only move to the 4 nearest neighboring grid points (the von Neumann Neighborhood of radius 1 [11]). The probability of moving to a neighboring grid point is determined by the mobility model of interest. In the following, we present the two main components of the proposed Markov chain: state probabilities and transition probabilities.

In our model, the states are defined as [(Current Location); (Previous Location)] and Fig. 1 illustrates the potential states for a $3 \times 3$ grid. Depending on the location, the number of associated states is different. Observe from Fig. 1 that if the current location is at a corner, boundary, or middle grid point, there are 2, 3, and 4 associated states, respectively. The arrows in the figure represent potential transitions between the states.

As an example, Fig. 2 shows the state transitions for the state $[(1,1) ;(0,1)]$ in more detail, where $P_{F}, P_{B}, P_{L}$, and $P_{R}$ are the probabilities to move forward, backward, left, and right, respectively. Since the previous location is given to be $(0,1)$, there can be a transition from all 3 associated states of location $(0,1)$ to $[(1,1) ;(0,1)]$. For this state, the corresponding forward direction from $[(1,1) ;(0,1)]$ is toward $(2,1)$, then left direction is toward $(1,0)$, right direction is toward $(1,2)$, and finally, backward direction is $(0,1)$.

We denote the steady state probabilities of this Markov chain by $\pi=\left[\pi_{(i, j ; k, l)}\right]$ and the transition probability matrix by $\mathbf{T}$, where the entries of the matrix are the transition probabilities between the states $[(i, j) ;(k, l)]$. Accordingly, we

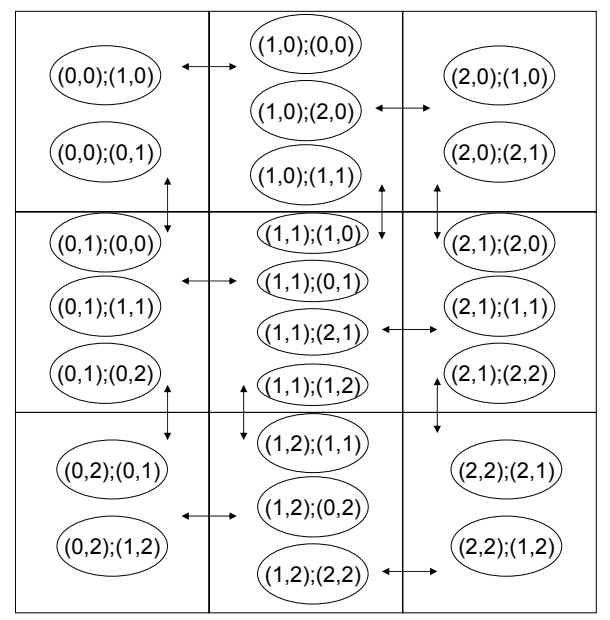

Fig. 1. The potential states of the Markov-chain for a $3 \times 3$ grid

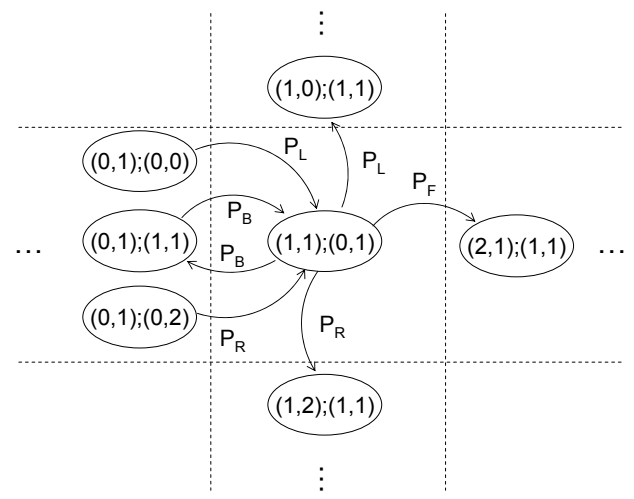

Fig. 2. State transition example for state $[(1,1) ;(0,1)]$

denote the transient state probabilities by $\pi^{(n)}=\left[\pi_{i, j: k, l}^{(n)}\right]$, at time step $n$. Then, we can write the following well-known relations for the steady-state and transient state probabilities [12]:

$$
\begin{aligned}
\pi & =\pi \mathbf{T} & \text { (for steady-state) } \\
\pi^{(n)} & =\pi^{(0)} \mathbf{T}^{n} & \text { (for transient state) } \\
\lim _{n \rightarrow \infty} \pi^{(n)} & =\pi &
\end{aligned}
$$

where $\sum \pi_{(i, j ; k, l)}=1$ and without loss of generalization the initial-state $\pi^{(0)}$ can be chosen to be $[1,0, \ldots, 0]$ (since the solution for $\pi$ is independent of the initial condition). From these linear equations, we can obtain the steady and transient state probabilities, which will be used to determine the coverage of a given mobility pattern.

\section{B. Coverage Metrics}

We denote the steady state coverage probability distribution for an $a \times a$ grid by $\mathbf{P}=[P(i, j)], 1 \leq i \leq a, 1 \leq j \leq a$. This probability matrix represents the percentage of time a given location $(i, j)$ is occupied and can be computed by adding the corresponding steady state probabilities obtained from (1):

$$
P(i, j)=\sum_{k, l} \pi_{(i, j ; k ; l)},
$$


where $(k, l)=\{(i-1, j),(i, j-1),(i+1, j),(i, j+1)\}$ for the non-boundary states. The $(k, l)$-pairs for boundary-states can be determined in a straightforward manner.

The transient coverage probability distribution, $\mathbf{P}^{(n)}=$ $\left[P^{(n)}(i, j)\right]$, is computed similarly as:

$$
P^{(n)}(i, j)=\sum_{k, l} \pi_{(i, j ; k ; l)}^{(n)}
$$

Using the obtained $\mathbf{P}^{(n)}$, we can compute the probability that location $(i, j)$ is covered by time step $n$ as follows:

$$
C^{(n)}(i, j)=1-\prod_{\nu=0}^{n}\left(1-P^{(\nu)}(i, j)\right)
$$

In the case of multiple drones, the state probabilities can easily be computed. Given the steady-state coverage distribution matrix of the drone $k$ is $\mathbf{P}_{k}$ (entries obtained using (2)) and assuming independent/decoupled mobility, the steady-state coverage distribution of an $m$-drone network can be obtained as:

$$
P^{\text {multi }}(i, j)=1-\prod_{k=1}^{m}\left(1-P_{k}(i, j)\right)
$$

The transient behavior of the $m$-drone network can be computed similarly, by substituting the $(i, j)$-th entry of the transient coverage probability matrix $\left(\mathbf{P}_{k}^{(n)}\right)$ (from (3)) into (5).

We now define some potential metrics of interest besides the coverage distribution of a mobility model in a grid: average coverage $\left(E\left\{C^{(n)}\right\}\right)$ and full coverage probability $\left(\xi^{(n)}\right)$ at time step $n$ for a grid of size $a \times a$ :

$$
\begin{aligned}
E\left\{C^{(n)}\right\} & =\frac{\sum_{i, j} C^{(n)}(i, j)}{a^{2}} \\
\xi^{(n)} & =\operatorname{Pr}\left(\mathbf{C}^{(n)}=\mathbf{1}_{a x a}\right)=\prod_{i, j} C^{(n)}(i, j)
\end{aligned}
$$

where $\mathbf{1}_{a x a}$ is an $a \times a$ matrix of ones.

These metrics carry some valuable information regarding the coverage performance, e.g., how well a given point is covered, how well the whole area is covered, or how much time would be necessary to cover the whole area.

In the next section, we provide the corresponding state transition probabilities for some representative independent and cooperative mobility models.

\section{Transition Probabilities}

\section{A. Independent Mobility}

In this section, we first shortly provide the state transition probabilities for the well-known random walk and random direction mobility models, where the transition probabilities are very intuitive. Note that for random walk the knowledge of the previous location is not necessary. Therefore, the states of the analytical tool $(i, j ; k, l)$ can be further simplified to $(i, j)$, however, we omit this step for consistency with the other models. For random walk, we assume that at each time step, the drone can go to any one of the neighboring grid points with equal probability. Clearly, the number of neighboring points change depending on the location (see Fig. 3 for a representation of the different areas). On the other hand, for random direction model, the direction is changed only when the drone reaches the boundary of the grid. Therefore, the previous location, which is also equivalent to direction for the lattice, needs to be taken into account. For both of these schemes as well as the cooperative scheme proposed in the next section, at the boundaries and corners the next location is chosen randomly among the available neighboring points with equal probability. Table I shows the forward, backward, left, and right transition probabilities for random walk and direction models, respectively. The entries are organized as [transition probability, location, direction of movement].

\begin{tabular}{|l|l|l|}
\hline $\mathbf{C}_{1}$ & $\mathbf{B}_{2}$ & $\mathbf{C}_{2}$ \\
\hline $\mathbf{B}_{1}$ & $\mathbf{M}$ & $\mathbf{B}_{3}$ \\
\hline $\mathbf{C}_{4}$ & $\mathbf{B}_{4}$ & $\mathbf{C}_{3}$ \\
\hline
\end{tabular}

Fig. 3. Location classification: corner $\left(C_{i}\right)$, boundary $\left(B_{i}\right)$, and middle $(M)$

TABLE I

RANDOM WALK (RW) AND DIRECTION (RD)

\begin{tabular}{|c||c|c|c|c|}
\hline & Corners & Boundaries & $\begin{array}{c}\text { Middle } \\
\text { RW }\end{array}$ & $\begin{array}{c}\text { Middle } \\
\text { RD }\end{array}$ \\
\hline \hline$P_{B}$ & $1 / 2\left(C_{i} \uparrow \rightarrow \downarrow \leftarrow\right)$ & $1 / 3\left(B_{i} \uparrow \rightarrow \downarrow \leftarrow\right)$ & $1 / 4$ & 0 \\
\hline$P_{F}$ & $0\left(C_{i} \uparrow \rightarrow \downarrow \leftarrow\right)$ & $\begin{array}{c}1 / 3\left(B_{i=1,3} \uparrow \downarrow,\right. \\
\left.B_{i=2,4} \leftarrow \rightarrow\right)\end{array}$ & $1 / 4$ & 1 \\
\hline$P_{L}$ & $\begin{array}{c}1 / 2\left(C_{1} \leftarrow, C_{2} \uparrow,\right. \\
\left.C_{3} \rightarrow, C_{4} \downarrow\right)\end{array}$ & $\begin{array}{c}1 / 3\left(B_{1} \leftarrow \downarrow, B_{2} \uparrow \leftarrow,\right. \\
\left.B_{3} \rightarrow \uparrow, B_{4} \downarrow \rightarrow\right)\end{array}$ & $1 / 4$ & 0 \\
\hline$P_{R}$ & $\begin{array}{c}1 / 2\left(C_{1} \uparrow C_{2} \rightarrow,\right. \\
\left.C_{3} \downarrow, C_{4} \leftarrow\right)\end{array}$ & $\begin{array}{c}1 / 3\left(B_{1} \leftarrow \uparrow, B_{2} \uparrow \rightarrow,\right. \\
\left.B_{3} \rightarrow \downarrow, B_{4} \downarrow \leftarrow\right)\end{array}$ & $1 / 4$ & 0 \\
\hline
\end{tabular}

\section{B. Cooperative Mobility}

In this section, we propose a method to approximate the coverage performance of a cooperative mobile network. In such a network, the nodes interact with each other (i.e., exchange information) whenever they meet. The amount or content of exchanged information is not within the scope of this paper. The objective is to come up with an appropriate transition probability matrix that can be used by the proposed stochastic tool. Recall that the proposed Markov chain is for a single drone. For independent mobility, it can easily be extended to multiple drones. However, for cooperative mobility this Markov chain is not sufficient to model the interactions. The states of a Markov-chain that exactly models all the interactions would grow exponentially with the number of drones. Therefore, in this paper, we propose an approximate method to model the behavior of the drones in a way that would allow us to treat the cooperative mobility as independent mobility.

To "decouple" the actions of the drones from each other we 
define the following for an $m$-drone network:

$$
P_{X}=\sum_{k=0}^{m-1} P_{X \mid k} \operatorname{Pr}(k+1 \text { nodes meet }), X \in\{B, F, L, R\}
$$

where the backward, forward, left-turn and right-turn probabilities are given by the decision metric $\left(P_{X \mid k}\right)$ of the cooperative mobility as well as the number of drones that meet. Clearly, probability of a meeting depends on the mobility model. However, for simplicity, in this work, we make the strong assumption that any node can be anywhere in the grid with equal probability. The implications of such an approximation will later be quantified by simulations. With this assumption, from the perspective of a drone at location $(i, j)$ of a grid of size $(a \times a)$, probability that exactly $k$ other nodes out of a total of $m$ drones will also be at $(i, j)$ is given by the binomial distribution:

$$
\operatorname{Pr}(\mathrm{k}+1 \text { nodes meet })=\left(\begin{array}{c}
m-1 \\
k
\end{array}\right)\left(\frac{1}{a^{2}}\right)^{k}\left(1-\frac{1}{a^{2}}\right)^{m-1-k}
$$

The entries of the corresponding transition probability matrix can then be computed using (7) and (8), given the decision metric $\left(P_{X \mid k}\right)$. If you have a cooperative rule quantified by decision metric $P_{X \mid k}$, these equations along with the analytical model from Section III can be used to quantify the coverage performance.

In the following, we provide an application of this method for simple cooperative mobility. It uses only the previous locations and number of the meeting drones in the decision criteria (e.g., as in [13]). The objective is to cover a given area as fast and as efficiently as possible. With such an objective, an intuitive rule is that the drones move to a previously unoccupied location with a high probability. Clearly, since we consider only the previous direction, the final decision might be good only locally and might not contribute to global coverage. The mobility rules at a grid point $(i, j)$ is summarized in Algorithm 1, where $n_{0}$ denote the number of unoccupied neighbors of $(i, j)$ at the previous time step.

Next, we derive the transition probabilities for the middle grid points. Observe that due to the symmetry of the decisions $P_{F \mid k}=P_{L \mid k}=P_{R \mid k}$, when $k \geq 1$. Therefore, if we compute $P_{B \mid k}$, all other probabilities would be determined as well. From the rules above, $P_{B \mid k}$ is non-zero only when $n_{0} \leq 1$. To this end, we first compute the probability that $n_{0}=1$ and $n_{0}=0$ given $k+1$ nodes meet.

There are $4^{k+1}$ different ways that $k+1$ nodes can meet. Assume that each of these meetings happen with equal probability $\frac{1}{4^{k+1}}$. Using combinatorics for selection with repetitions, we can derive the probabilities that $n_{0}=1$ and $n_{0}=0$, respectively, when $k+1$ nodes meet as follows:

$$
\operatorname{Pr}\left(n_{0}=1\right)=\frac{\sum_{i=1}^{k-1} \sum_{j=1}^{k-i}\left(\begin{array}{c}
k+1 \\
i
\end{array}\right)\left(\begin{array}{c}
k+1-i \\
j
\end{array}\right)}{4^{k}}
$$

and

$$
\begin{aligned}
& \operatorname{Pr}\left(n_{0}=0\right)= \\
& \frac{\sum_{i=1}^{k-2} \sum_{j=1}^{k-i-1} \sum_{l=1}^{k-i-j}\left(\begin{array}{c}
k+1 \\
i
\end{array}\right)\left(\begin{array}{c}
k+1-i \\
j
\end{array}\right)\left(\begin{array}{c}
k+1-i-j \\
l
\end{array}\right)}{4^{k+1}} .
\end{aligned}
$$

\begin{tabular}{l} 
Algorithm 1 Cooperative Mobility Algorithm \\
\hline 1) If $(i, j)$ is not in the boundaries or corners of the grid:
\end{tabular}

a) If $k=0$, i.e., there is only one drone at $(i, j)$, the drone keeps going forward until it meets another drone or until it hits the boundary.

b) If $k \geq 1$, the drones determine the unoccupied neighbors, $n_{0}$, of $(i, j)$ at the previous time step and

i) If $n_{0} \geq 2$, then the drones move to any one of the unoccupied grid points with probability $\frac{1}{n_{0}}$

ii) If $n_{0}=1$, then the drones move to the unoccupied grid point with probability $p_{0}$ and the other 3 occupied grid points with probability $\frac{1-p_{0}}{3}$. Clearly, if $p_{0}=1$, this rule is equivalent to above rule $(i)$. A non-zero $p_{0}$ option is given to prevent all drones from moving into the same location.

iii) If $n_{0}=0$, then the drones move any one of the 4 neighboring grid points with probability $1 / 4$.

2) If $(i, j)$ is in one of the boundaries or corners of the grid, then the same rules as the independent random mobility models are applied regardless of the presence of a meeting.

Table II presents these probabilities for different $k$ values. As the number of nodes that meet, i.e., $k+1$ increases, as expected, $\operatorname{Pr}\left(n_{0}=0\right)$ increases and correspondingly, $\operatorname{Pr}\left(n_{0}=\right.$ 1) starts decreasing after a certain point.

TABLE II

$$
\operatorname{Pr}\left(n_{0}=0\right) \text { AND } \operatorname{Pr}\left(n_{0}=1\right)
$$

\begin{tabular}{|c||c|c|c|c|c|c|}
\hline & $k=1$ & $k=2$ & $k=3$ & $k=4$ & $k=5$ & $k=6$ \\
\hline \hline $\operatorname{Pr}\left(n_{0}=0\right)$ & 0 & 0 & 0.09 & 0.23 & 0.38 & 0.51 \\
\hline $\operatorname{Pr}\left(n_{0}=1\right)$ & 0 & 0.375 & 0.56 & 0.59 & 0.53 & 0.44 \\
\hline
\end{tabular}

Using (9) and (10), $P_{B \mid k}$ for $m>3$ is given by:

$$
\begin{aligned}
& P_{B \mid k}=\sum_{i=0}^{3} \operatorname{Pr}\left(n_{0}=i\right) P_{B \mid k i} \\
& = \begin{cases}0, & k<2 \\
\operatorname{Pr}\left(n_{0}=1\right) \frac{1-p_{0}}{3}, & k=2 \\
\operatorname{Pr}\left(n_{0}=1\right) \frac{1-p_{0}}{3}+\operatorname{Pr}\left(n_{0}=0\right) \frac{1}{4}, & 2<k \leq m-1\end{cases}
\end{aligned}
$$

When $m<3, P_{B \mid k}=0$, and when $m=3, P_{B \mid k}=\operatorname{Pr}\left(n_{0}=\right.$ 1) $\frac{1-p_{0}}{3}$.

Finally, substituting (8) and (11) into (7) we can compute $P_{B}$. Similarly, $P_{F}, P_{R}$, and $P_{L}$ can be computed substituting the following relations into (7):

$$
P_{F \mid k}= \begin{cases}1, & k=0 \\ \frac{1-P_{B \mid k}}{3}, & 0<k \leq m-1\end{cases}
$$

and

$$
P_{R \mid k}=P_{L \mid k}= \begin{cases}0, & k=0 \\ \frac{1-P_{B \mid k}}{3}, & 0<k \leq m-1 .\end{cases}
$$


Then, the transition probability matrix for each drone can be obtained using the derived $P_{X}$ 's for the middle cells and the boundary/corner grid behavior described in the previous subsection. The overall coverage performance of an $m$-drone cooperative network can then be determined using (5).

\section{Coverage Performance and its Discussion}

In this section, we evaluate the validity of the proposed analytical method for several different scenarios by comparing the analysis with Monte Carlo simulations (where the coverage distributions are obtained by averaging over 10000 runs). For the cooperative mobility model, we use $p_{0}=0.25$. Different number of drones $(m)$, grid sizes $(a \times a)$, and time steps $(n)$ are evaluated.

First, we evaluate the steady-state coverage distribution, which corresponds to the percentage of time a given point would be covered. Fig. 4 (a) and (b) show the average time coverage versus number of drones $m$ and the grid dimension $a$. The steady-state coverage distribution matrix is computed using (1) and (2). Both the analytical and simulation results in the figure are then obtained by averaging overall points in the grid. Observe that the steady-state performance of all schemes are the same, shown by both simulation and analysis. While the limiting distributions of all the schemes are the same, the time required to reach this distribution varies between mobility models.

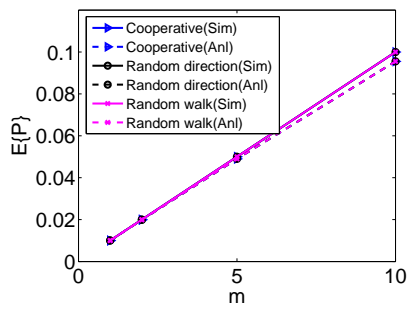

(a) $\mathrm{E}\{\mathbf{P}\}$ versus $m, a=10$

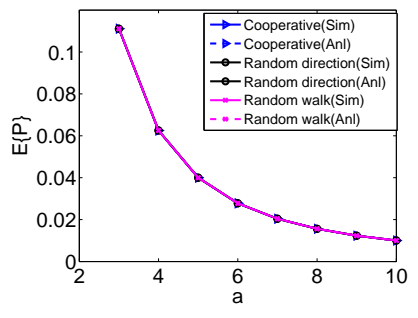

(b) $\mathrm{E}\{\mathbf{P}\}$ versus $a, m=10$
Fig. 4. Average steady-state (time) coverage.

Next, we look at the transient behavior of the mobility models under study. Fig. 5 shows a snapshot of the coverage at time step $n=10$ for different number of drones and grid dimensions. As expected, the coverage increases with increasing number of drones and decreases with grid size. While for $a=5$ coverage over $90 \%$ can be achieved with 10 drones, the achievable coverage drops below $40 \%$ when the grid size is increased to $10 \times 10$. Observe that the simulation and analysis results are in agreement in general. The highest deviation is observed as the number of drones increase. Recall that the average coverage is computed over all grid points, and hence, the deviations in the coverage of each grid point, however small they maybe, propagate and could become significantly large when added. Therefore, to check the similarity of the coverage probability distributions obtained from the analysis and the simulation, we use the following Euclidean distance metric:

$$
M S E^{(n)}=E\left\{\left(C_{a n l}^{(n)}(i, j)-C_{\text {sim }}^{(n)}(i, j)\right)^{2}\right\} .
$$

Fig. 6 presents the mean square error obtained using (14), when $n=10$ and $a=10$. Observe from these results that while the average coverage obtained from the analysis and simulation may deviate from each other, the individual coverage of the grid points on average deviate around $0.18 \%$. We are currently in the process of determining a distance metric that does not suffer from numerical approximation limitations to better identify the deviations.

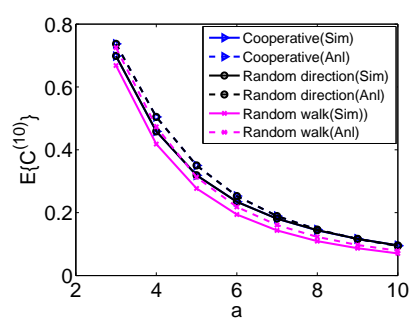

(a) $\mathrm{E}\left\{C^{(10)}\right\}$ versus $a, m=1$

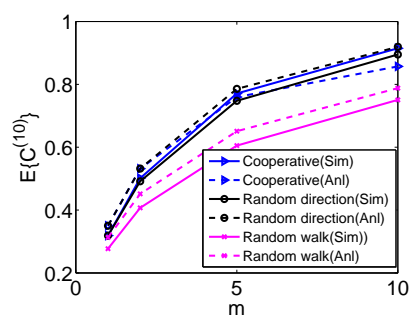

(c) $\mathrm{E}\left\{C^{(10)}\right\}$ versus $m, a=5$

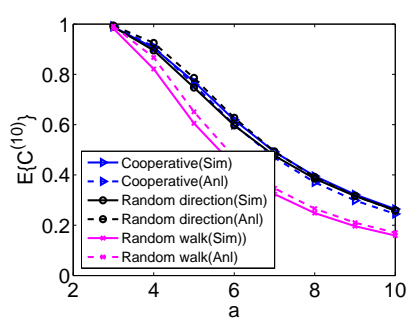

(b) $\mathrm{E}\left\{C^{(10)}\right\}$ versus $a, m=5$

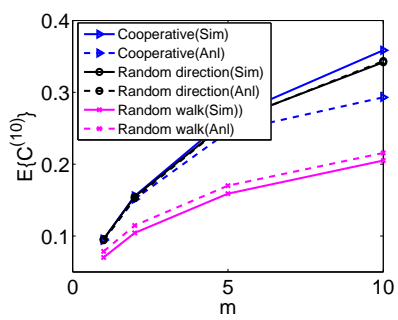

(d) $\mathrm{E}\left\{C^{(10)}\right\}$ versus $m, a=10$
Fig. 5. Average spatial coverage when $n=10$ steps.

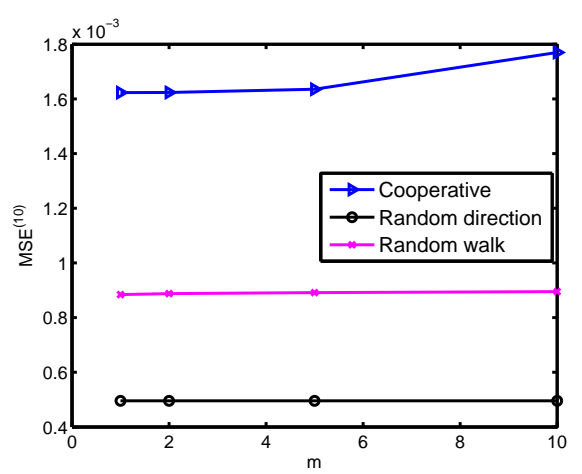

Fig. 6. MSE versus $m$, when $n=10$ and $a=10$.

Finally, we illustrate the progress of coverage with time. Fig. 7 shows the MSE, average and full spatial coverages (from (6)), when $a=5$ and $m=\{1,5\}$. The MSE reduces as the number of time steps increase and is less than $2 \%$ for $m=1$ and less than $0.4 \%$ when $m=5$. As a result, the average and full spatial coverages from analysis and simulation also deviate from each other less, when $m=5$. Comparing average and full coverages, we observe that while the likelihood that each point is covered on average can be above $99 \%$ around $n=200$ (when $m=1$ ), full coverage requires significantly more time. Therefore, a threshold-based coverage metric can be more suitable than average or full coverage for some applications. Nevertheless, the analytical tool can provide some insight 
into how much time would be required to achieve a certain coverage level and allows for testing different performance metrics of interest.
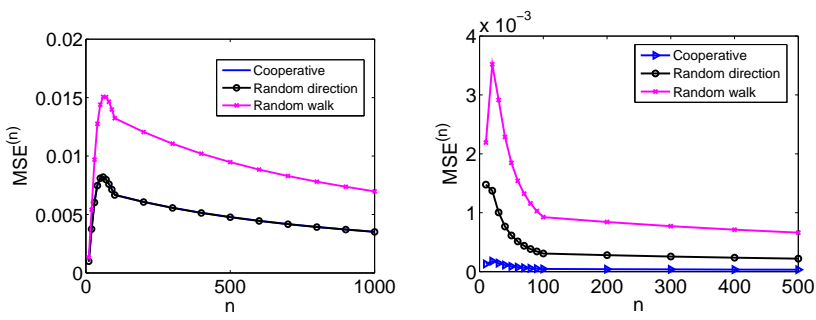

(a) MSE versus $n$, when $a=5$ and $m=1$ (left), $m=5$ (right)
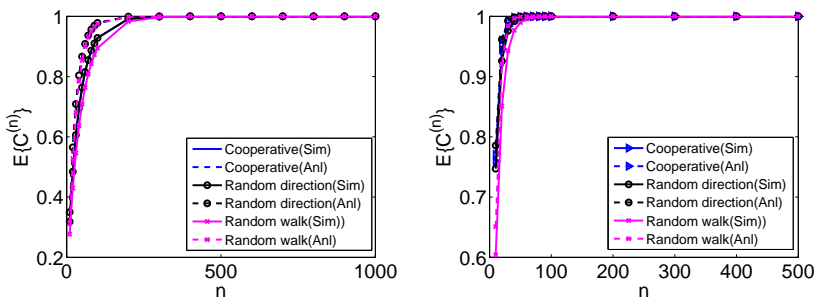

(b) $\mathrm{E}\left\{C^{(n)}\right\}$ versus $n$, when $a=5$ and $m=1$ (left), $m=5$ (right)
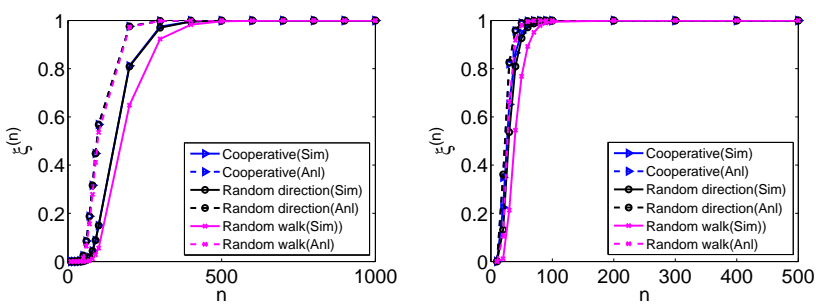

(c) $\xi^{(n)}$ versus $n$, when $a=5$ and $m=1$ (left), $m=5$ (right)

Fig. 7. Transient behavior comparison

\section{CONCLUSiOnS}

In this work, we proposed an analytical model to evaluate the coverage performance of a networked UAV system. We showed the validity and the limitations of the analytical tool by comparing with simulations for several scenarios. The performance metrics of interest focused on in this paper were coverage distribution, average and full coverages. We observed that while the coverage distributions can be estimated well with the analytical model, the average and full coverages can deviate from the simulations for certain system parameters due to error propagation.

\section{ACKNOWLEDGMENT}

This work was performed in the project Collaborative Microdrones (cDrones) of the research cluster Lakeside Labs and was partly funded by the European Regional Development Fund, the Carinthian Economic Promotion Fund (KWF), and the state of Austria under grant 20214/17095/24772.

\section{REFERENCES}

[1] E. Kuiper and S. Nadjm-Tehrani, "Mobility models for uav group reconnaissance applications," in Proc. Intl. Conf. Wireless and Mobile Communications (ICWMC), 2006.
[2] M. Grossglauser and D. Tse, "Mobility increases the capacity of adhoc wireless networks," IEEE/ACM Trans. on Networking, vol. 10, pp. 477-486, 2001.

[3] D. K. Goldenberg, J. Lin, A. S. Morse, B. E. Rosen, and Y. R. Yang, "Towards mobility as a network control primitive," in Proc. ACM Intl. Symp. on Mobile Ad hoc Networking and Computing (MobiHoc), 2004, pp. 163-174.

[4] B. Liu, P. Brass, O. Dousse, P. Nain, and D. Towsley, "Mobility improves coverage of sensor networks," in Proc. ACM Intl. Symp. Mob. Ad hoc Net. Comp. (MobiHoc), 2005, pp. 300-308.

[5] G. Lin, G. Noubir, and R. Rajaraman, "Mobility models for ad hoc network simulation," in Proc. IEEE Conf. Comp. (INFOCOM), vol. 1, 2004, pp. 454-463.

[6] D. Engelhart, A. Sivasubramaniam, C. Barrett, M. Marathe, J. Smith, and M. Morin, "A spatial analysis of mobility models: application to wireless ad hoc network simulation," in Proc. Simulation Symp., 2004, pp. $3-42$.

[7] L. E. Parker, "Distributed algorithms for multi-robot observation of multiple moving targets," Autonomous Robots, vol. 12, no. 3, pp. 231255, 2002.

[8] M. Ahmadi and P. Stone, "A multi-robot system for continuous area sweeping tasks," in Proceedings of International Conference on Robotics and and Automation (ICRA'06), 2006, p. 17241729.

[9] A. Elfes, "Using occupancy grids for mobile robot perception and navigation," Computer, vol. 22, no. 6, pp. 46-57, June 1990.

[10] J. Leonard and H. Durrant-Whyte, "Simultaneous map building and localization for an autonomous mobile robot," in Proc. IEEE/RSJ Intl. Workshop on Intelligent Robots and Systems (IROS), vol. 3, Nov. 1991, pp. 1442-1447.

[11] L. Gray, "A mathematician looks at Wolfram's new kind of science," Notices of the AMS, vol. 50, no. 2, pp. 200-211, Feb. 2003.

[12] L. Kleinrock, Queueing Systems Volume I: Theory. New York: Wiley, 1975.

[13] E. Yanmaz and C. Bettstetter, "Area coverage with unmanned vehicles: A belief-based approach," in Proc. IEEE Vehicular Technology Conf. (VTC), 2010, pp. 1-5 (CD). 